\title{
ON THE SEMICOMPLEXES OF F. BROWDER
}

\author{
BY RICHARD B. THOMPSON ${ }^{1}$
}

Communicated by Felix Browder, January 30, 1967

1. Introduction. In [4] Lefschetz defined quasi-complexes and proved his fixed point theorem for that class of spaces. Since then, further discussions of quasi-complexes have appeared in [1], [3] and [6]. Unfortunately, the question of whether or not the class of quasicomplexes contains the class of compact metric ANR's has never been settled. In [2] F. Browder in troduced the concept of semicomplexes and showed that this class does contain the compact metric ANR's as well as admitting a local fixed point index for continuous maps. In $\$ 2$ of this announcement the relationship between these two concepts is clarified by defining weak semicomplexes and showing that this class contains both the quasi-complexes and the semicomplexes. Furthermore, the Lefschetz fixed point theorem holds for weak semicomplexes and within this class the subclasses of quasi-complexes and semicomplexes are completely characterized. In $\S \S 3,4$ and 5 new results on the existence and uniqueness of semicomplexes are given.

The proofs of these and additional results will appear elsewhere. The author wishes to thank Professor Edward Fadell for suggesting several of the questions which are considered in this work.

2. Weak semicomplexes. The definition of a weak semicomplex is motivated by the following definition of a semicomplex which is a slight modification of that given by Browder in [2]. This definition is, however, sufficient for use in all of Browder's results and proofs.

If $X$ is a compact Hausdorff space, $\Sigma(X)$ will denote the set of all finite covers of $X$ by open sets. If $\alpha \in \Sigma(X)$ then $N_{\alpha}$ will stand for the nerve of $\alpha$ and $C\left(N_{\alpha}\right)$ will stand for the associated chain complex of $N_{\alpha}$ with rational coefficients. When $\alpha$ and $\beta$ are in $\Sigma(X)$ with $\beta$ refining $\alpha$ (i.e., $\beta>\alpha$ ), $\pi_{\alpha}^{\beta}$ : $C\left(N_{\beta}\right) \rightarrow C\left(N_{\alpha}\right)$ will denote the usual chain map induced by a vertex transformation based on set inclusion.

Definition (1). A semicomplex, $S(X)=\left\{X, g, \Omega, \alpha_{0}, C_{\lambda}\right\}$, is a quintuple where $X$ is a compact Hausdorff space; $\mathscr{I}$ is a collection of finite covers of $X$ by connected open sets which is cofinal in $\Sigma(X) ; \Omega$ is a cofinal subset of $\Sigma(X) ; \alpha_{0}$ is a function from $\mathscr{I}$ into $\Omega$ such that for each $\lambda \in \mathscr{I}, \alpha_{0}(\lambda)>\lambda$; and $C$ is a function assigning to $\lambda \in \mathscr{I}$ a family,

${ }_{1}^{1}$ This research was supported by the National Science Foundation under grant GP-6682 and is part of the author's doctoral dissertation written under the direction of Professor Edward Fadell. 
$C_{\lambda}$, of chain maps consisting of one or more chain maps $c_{\alpha}^{\beta}: C\left(N_{\beta}\right)$ $\rightarrow C\left(N_{\alpha}\right)$ for every pair $\alpha, \beta \in \Omega$ such that $\alpha>\beta>\alpha_{0}(\lambda)$. These chain maps $c_{\alpha}^{\beta}$ are called antiprojections and are assumed to preserve the Kronecker index as well as satisfy the following axioms.

(i) If $\alpha>\beta>\gamma>\alpha_{0}(\lambda), \alpha, \beta, \gamma \in \Omega$ and $c_{\alpha}^{\beta}, c_{\alpha}^{\gamma} \in C_{\lambda}$ then there exists a chain homotopy $\Delta_{\alpha}^{\beta}$ connecting $c_{\alpha}^{\beta}$ and $c_{\alpha}^{\gamma} \pi_{\gamma}^{\beta}$ such that for each $\sigma \in N_{\beta}$ there is a set $U \in \lambda$ with $\sup (\sigma) \cup \sup \left(c_{\alpha}^{\beta}(\sigma)\right) \cup \sup \left(\Delta_{\alpha}^{\beta}(\sigma)\right) \subseteq U$.

(ii) If $\alpha>\beta>\gamma>\alpha_{0}(\lambda), \alpha, \beta, \gamma \in \Omega$ and $c_{\beta}^{\gamma}, c_{\alpha}^{\gamma} \in C_{\lambda}$ then there exists a chain homotopy $\Gamma_{\beta}^{\gamma}$ connecting $c_{\beta}^{\gamma}$ and $\pi_{\beta}^{\alpha} c_{\alpha}^{\gamma}$ such that for each $\sigma \in N_{\gamma}$ there is a set $V \in \lambda$ with $\sup (\sigma) \cup \sup \left(c_{\beta}^{\gamma}(\sigma)\right) \cup \sup \left(\Gamma_{\beta}^{\gamma}(\sigma)\right) \subseteq V$.

(iii) If $\alpha>\alpha_{0}(\lambda), \alpha \in \Omega$ and $c_{\alpha}^{\alpha} \in C_{\lambda}$, then $c_{\alpha *}^{\alpha}: H\left(N_{\alpha} ; Q\right) \rightarrow H\left(N_{\alpha} ; Q\right)$ is an idempotent endomorphism whose image is exactly the image of the projection homomorphism $p_{\alpha}: H(X ; Q) \rightarrow H\left(N_{\alpha} ; Q\right)$ where $H(X ; Q)$ denotes Cech homology with rational coefficients.

(iv) If $\mu>\lambda$ then $\alpha_{0}(\mu)>\alpha_{0}(\lambda)$ and if $\alpha>\beta>\alpha_{0}(\mu), \alpha, \beta \in \Omega, c_{\alpha}^{\beta}(\mu) \in C_{\mu}$ and $c_{\alpha}^{\beta}(\lambda) \in C_{\lambda}$, then there exists a chain homotopy $\theta_{\alpha}^{\beta}$ connecting these two antiprojections such that for each $\sigma \in N_{\beta}$ there is a set $W \in \lambda$ with $\sup (\sigma) \cup \sup \left(c_{\alpha}^{\beta}(\lambda)(\sigma)\right) \cup \sup \left(\theta_{\alpha}^{\beta}(\sigma)\right) \subseteq W$.

For convenience $S(X)$ will sometimes be called a semicomplex structure on the underlying space $X$.

If less stringent conditions are placed on the antiprojections, an interesting theory can still be developed.

Definition (2). A weak semicomplex $S(X)=\{X, \Omega, C\}$ is a triple where $X$ is a compact Hausdorff space; $\Omega$ is a function assigning to each $\lambda \in \Sigma(X)$ a cofinal subset $\Omega_{\lambda}$ of $\Sigma(X)$ which has a designated coarsest element $\alpha_{0}(\lambda)$ such that $\alpha_{0}(\lambda)>\lambda$; and $C$ is a function assigning to each $\lambda \in \Sigma(X)$ a family, $C_{\lambda}$, of chain maps consisting of one or more chain maps $c_{\alpha}^{\beta}: C\left(N_{\beta}\right) \rightarrow C\left(N_{\alpha}\right)$ for every pair $\alpha, \beta \in \Omega_{\lambda}$ such that $\alpha>\beta>\alpha_{0}(\lambda)$. Each $c_{\alpha}^{\beta} \in C_{\lambda}$ has the property that if $\sigma \in N_{\beta}$ then there is a set $U \in \lambda$ with $\sup (\sigma) \cup \sup \left(c_{\alpha}^{\beta}(\sigma)\right) \subseteq U$. These chain maps are called antiprojections and are assumed to satisfy the following axioms.

(i) If $\alpha>\beta>\gamma>\alpha_{0}(\lambda), \alpha, \beta, \gamma \in \Omega_{\lambda}$ and $c_{\alpha}^{\beta}, c_{\alpha}^{\gamma} \in C_{\lambda}$ then $c_{\alpha}^{\beta}$ is chain homotopic $(\sim)$ to $c_{\alpha}^{\gamma} \pi_{\gamma}^{\beta}$.

(ii) If $\alpha>\beta>\gamma>\alpha_{0}(\lambda), \alpha, \beta, \gamma \in \Omega_{\lambda}$ and $c_{\beta}^{\gamma}, c_{\alpha}^{\gamma} \in C_{\lambda}$ then $c_{\beta}^{\gamma} \sim \pi_{\beta}^{\alpha} c_{\alpha}^{\gamma}$.

(iii) If $\alpha>\alpha_{0}(\lambda), \alpha \in \Omega_{\lambda}$ and $c_{\alpha}^{\alpha} \in C_{\lambda}$ then $c_{\alpha *}^{\alpha}: H\left(N_{\alpha} ; Q\right) \rightarrow H\left(N_{\alpha} ; Q\right)$ is an idempotent endomorphism whose image is exactly the image of the projection homomorphism $p_{\alpha}: H(X ; Q) \rightarrow H\left(N_{\alpha} ; Q\right)$.

Definition (3). A weak semicomplex, $\{X, \Omega, C\}$, is called simple if for each $\lambda, \lambda \in \Sigma(X), \alpha \in \Omega_{\lambda}$ and $c_{\alpha}^{\alpha} \in C_{\lambda}, c_{\alpha}^{\alpha} \sim 1: C\left(N_{\alpha}\right) \rightarrow C\left(N_{\alpha}\right)$. 
Proposition (1). If a space $X$ has a weak semicomplex structure $S(X)=\{X, \Omega, C\}$ then for all $\lambda$ the projection homomorphism $p_{\alpha_{0}(\lambda)}: H(X ; Q) \rightarrow H\left(N_{\alpha_{0}(\lambda)} ; Q\right)$ is injective. If $S(X)$ is simple then $p_{\alpha_{0}(\lambda)}$ is also surjective.

A consequence of this proposition is that if $X$ has a weak semicomplex structure then $X$ is weakly locally connected in all dimensions in the sense that all rational Cech cycles of any dimension, which have sufficiently small support, bound on $X$. It is also clear in this case that $H(X ; Q)$ is finitely generated so that if $f: X \rightarrow X$ is a map then the Lefschetz number $L(f)$ is defined.

THEOREM (1). If $X$ has a weak semicomplex structure then for any fixed point free map $f: X \rightarrow X, L(f)=0$.

TheOREM (2). A space $X$ is a quasi-complex if and only if it has a simple weak semicomplex structure.

3. Categories of semicomplexes. It is clear from Definition (1) that if $\left\{X, g, \Omega, \alpha_{0}, C\right\}$ is a semicomplex then $X$ is forced to be locally connected.

Let $S(X)=\left\{X, \mathfrak{g}, \Omega, \alpha_{0}, C\right\}$ be a semicomplex. For each $\lambda \in \Sigma(X)$, pick a refinement $\lambda^{\prime} \in \mathscr{g}$ and a cofinal subset $\Omega_{\lambda}^{\prime}$ of $\left\{\alpha \in \Omega \mid \alpha>\alpha_{0}\left(\lambda^{\prime}\right)\right\}$ which has a designated coarsest element $\alpha_{0}^{\prime}(\lambda)$. Let $\Omega^{\prime}$ be the function that assigns to $\lambda$ the set $\Omega_{\lambda}^{\prime}$ and let $C^{\prime}$ be the function that assigns to $\lambda$ the family of chain maps $C_{\lambda}^{\prime}=\left\{c_{\beta}^{\alpha} \in C_{\lambda} \mid \alpha, \beta \in \Omega_{\lambda}^{\prime}\right\}$. Clearly, the derived structure $S^{\prime}(X)=\left\{X, \Omega^{\prime}, C^{\prime}\right\}$ is a weak semicomplex.

If $S(X)=\left\{X, \mathscr{J}, \Omega, \alpha_{0}, C\right\}$ and $S(Y)=\left\{Y, \mathscr{g}, \Psi, \beta_{0}, D\right\}$ are semicomplexes, a map $h: X \rightarrow Y$ is called a semicomplex map from $S(X)$ into $S(Y)$ if $h$ is compatible with these structures. The precise definition of this compatibility is quite technical but the basic idea is that there are arbitrarily fine covers $\alpha, \beta \in \Omega$ and $\gamma, \delta \in \Psi$ such that $h$ induces chain maps which make the following diagram chain homotopy commutative with a chain homotopy of small support on simplexes.

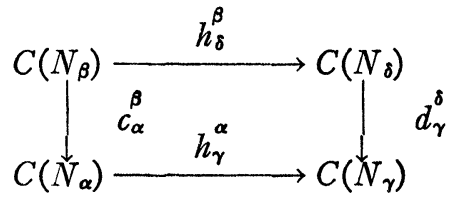

Browder's main result in [2] concerns the existence of a local fixed point index. 
ThEOREM (3). If $\mathfrak{C}$ is a category of semicomplexes and semicomplex maps then a local fixed point index exists for continuous self-maps on the underlying spaces of objects in $\mathfrak{C}$.

This particular index, which will be called the Browder index, appears to depend on the particular semicomplex structures involved and is clearly influenced by which of the continuous maps are semicomplex maps. To study this dependence an equivalence relation is introduced.

Definition (4). Two semicomplexes $S(X)$ and $T(X)$ are equivalent $(\approx)$ if the identity map $1_{X}$ on $X$ is as emicomplex map from $S(X)$ into $T(X)$.

Proposition $(2) . \approx$ is an equivalence relation on the collection of all semicomplexes.

If $\mathcal{C}$ is a category of semicomplexes one can use $\approx$ to define an equivalence relation among the semicomplex maps in $\mathcal{C}$. Thus a category $[\mathfrak{e}]$ can be defined with equivalence classes $[S(X)]$ and $[h]$ of the semicomplexes and semicomplex maps in $\mathcal{C}$ as objects and morphisms.

Proposition (3). The Browder index yields the same indices when applied to equivalent semicomplexes. Hence, the Browder index on a category $\mathrm{e}$ induces a local fixed point index on [e].

Finally, let $\mathfrak{e}^{\prime}$ be the category whose objects are all spaces which are the underlying space of some semicomplex in $\mathcal{C}$ and whose morphisms are all maps between these spaces.

Definition (5). A category $\mathcal{C}$ of semicomplexes and semicomplex maps is fully reducible if $[\mathfrak{C}]$ is isomorphic to $\mathfrak{C}^{\prime}$ under the forgetful functor taking $[S(X)] \rightarrow X$ and $[h] \rightarrow h$.

Proposition (4). If $\mathfrak{C}$ is a fully reducible category then a local fixed point index is defined on $\mathfrak{C}^{\prime}$ which does not depend in any way on the semicomplex structures used. In particular if $S(X)$ and $S(Y)$ are in $\mathfrak{e}$ then any map $f: X \rightarrow Y$ is a semicomplex map from $S(X)$ to $S(Y)$.

Definition (6). A semicomplex $S(X)=\left\{X, \mathfrak{g}, \Omega, \alpha_{0}, C\right\}$ is called simple if in addition to axioms (i)-(iv) of Definition (1) it satisfies the following axiom.

(v) If $\alpha>\alpha_{0}(\lambda), \alpha \in \Omega$ and $c_{\alpha}^{\alpha} \in C_{\lambda}$ then there exists a chain homotopy $\Lambda_{\alpha}^{\alpha}$ connecting $c_{\alpha}^{\alpha}$ and 1:C(N $) \rightarrow C\left(N_{\alpha}\right)$ such that for each $\sigma \in N_{\alpha}$ there is a set $W \in \lambda$ with $\sup (\sigma) \cup \sup \left(c_{\alpha}^{\alpha}(\sigma)\right) \cup \sup \left(\Lambda_{\alpha}^{\alpha}(\sigma)\right) \subseteq W$.

Proposition (5). If $S(X)=\left\{X, \mathfrak{g}, \Omega, \alpha_{0}, C\right\}$ is a simple semicomplex then any derived weak semicomplex $S^{\prime}(X)=\left\{X, \Omega^{\prime}, C^{\prime}\right\}$ is also simple. 
Thus the following relations exist among the various structures.

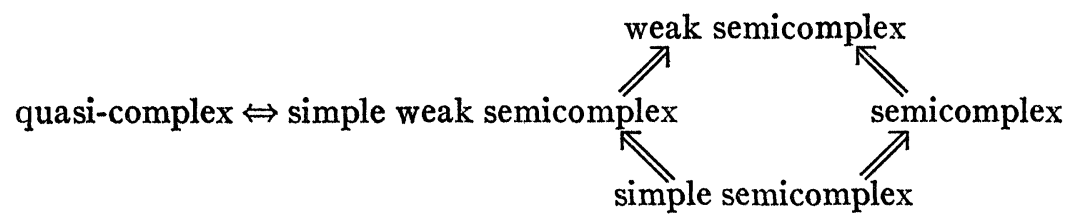

\section{Examples.}

Proposition (6). Every compact polyhedron has a simple semicomplex structure.

In [5] Lefschetz defined a class of compact spaces which he called HLC* spaces. These include the compact metric ANR spaces and the compact generalized manifolds of Wilder [7].

Proposition (7). Every metric HLC* space has a semicomplex structure.

THEOREM (4). $\S_{*}$, the category of all semicomplex structures and semicomplex maps on metric HLC* spaces, is a fully reducible category.

The proof of Theorem (4) relies heavily on the properties of the underlying HLC* spaces. However, if only simple semicomplexes are considered no assumptions need be made about the underlying spaces

THEOREM (5). $\varsigma_{S}$, the category of all simple semicomplexes and their semicomplex maps, is a fully reducible category.

It was shown in [3] and [6] that all snake-like continua and their hyperspaces are quasi-complexes and hence have simple weak semicomplex structures.

5. Products and Suspensions. Let $S$ be the set of all semicomplexes; $S_{S}$ the set of all simple semicomplexes; $S_{W}$ the set of all weak semicomplexes and $\delta_{S W}$ the set of all simple weak semicomplexes.

Theorem (6). If $\mathfrak{C}$ is any one of $\mathcal{S}, \mathcal{S}_{S}, \mathfrak{S}_{W}$ and $\mathfrak{S}_{S W}$ with $S(X)$ and $S(Y)$ in $\mathcal{C}$ then there exists a structure $S(X) \times S(Y)$ on the space $X \times Y$ which is in $\mathbb{C}$. Further, in the case of $S$, this is natural in the sense that if $h: S(X) \rightarrow S(Y)$ and $h^{\prime}: S\left(X^{\prime}\right) \rightarrow S\left(Y^{\prime}\right)$ are semicomplex maps with $S(X), S\left(X^{\prime}\right), S(Y)$, and $S\left(Y^{\prime}\right)$ in $s$ then $h \times h^{\prime}: S(X) \times S\left(X^{\prime}\right) \rightarrow S(Y)$ $\times S\left(Y^{\prime}\right)$ is a semicomplex map.

Theorem (7). If $\mathfrak{e}$ is any one of $\mathcal{S}, \mathcal{S}_{S}, \mathfrak{s}_{W}$ and $\mathfrak{s}_{S W}$ with $S(X)$ in $\mathfrak{e}$ then there exists a structure $\operatorname{Susp}(S(X))$ on the suspension of $X$ which is in e. Further, if $h: S(X) \rightarrow S(Y)$ is a semicomplex map with $S(X)$ 
and $S(Y)$ in $S$ then the suspension of $h$ is a semicomplex map from $\operatorname{Susp}(S(X))$ into $\operatorname{Susp}(S(Y))$.

\section{BIBLIOGRAPHY}

1. T. Brahana, Products of quasi-complexes, Proc. Amer. Math. Soc. 7 (1956), 954-958.

2. F. Browder, On the fixed point index for continuous mappings of locally connected spaces, Summa Brasiliensis Mathematicae 4 fasc. 7 (1960), 253-293.

3. E. Dyer, $A$ fixed point theorem, Proc. Amer. Math. Soc. 7 (1956), 662-672.

4. S. Lefschetz, Algebraic topology, Amer. Math. Soc. Colloq. Publ., vol. 27, Amer. Math. Soc., Providence, R. I., 1942.

5. - Topics in topology, Princeton Univ. Press, Princeton, New Jersey, 1942.

6. J. Segal, $A$ fixed point theorem for the hyperspace of a snake-like continuum, Fund. Math. 50 (1962), 237-248.

7. R. Wilder, Topology of manifolds, Rev. ed., Amer. Math. Soc. Colloq. Publ., vol. 32, Amer. Math. Soc., Providence, R. I., 1949.

UNIVERSITY OF WISCONSIN 